\title{
Heavy Flavor Mesons in Strong Magnetic Fields
}

\author{
Peter Filip ${ }^{* \dagger}$ \\ Institute of Physics, Slovak Academy of Sciences, Dúbravská cesta 9, Bratislava 84511, Slovakia \\ E-mail: Peter.Filipesavba.sk
}

\begin{abstract}
The response of heavy pseudoscalar and vector mesons to the strong magnetic fields created in heavy ion collisions is studied. Interaction of the constituent quark magnetic moments with the external magnetic field is described using the analogy of mesons with Positronium and Muonium bound states. Magnetic moments of open-flavor vector mesons are estimated and magnetic polarizability of pseudoscalar mesons is discussed. For heavy Quarkonium we predict a quantum mixing of spin singlet states $\eta_{c}, \eta_{b}$ with $\left(m_{z}=0\right)$ substates of $\mathrm{J} / \psi$ and $\Upsilon$ mesons, which results in the modification of their decay widths (lifetimes) and decay channels. We also consider the case of $\varphi(1020)$ meson. The influence of the Quarkonium states mixing on dilepton spectrum observed in heavy ion collisions is discussed.
\end{abstract}

8th International Workshop on Critical Point and Onset of Deconfinement (CPOD 2013)

March 11 - 15, 2013

Napa, California, USA

* Speaker.

${ }^{\dagger}$ With special thanks to organizers for the invitation. 


\section{Introduction}

Magnetic fields created in relativistic heavy ion collisions [1] are predicted to be extremely large: $B \approx 10^{15}$ Tesla. During their short-time existence, such fields can interact with the magnetic moments of quarks, influence properties of strongly interacting partonic matter [2] and possibly also change the internal structure of hadrons.

Quarks, having electric charge, color charge and weak-hypercharge, should have magnetic, chromo-magnetic and also weak-magnetic moments, as can be infered from Dirac equation [3]. Magnetic dipole moment is generated "automatically" around any charged massive body with the angular momentum, due to non-diagonal metric tensor $g^{\mu v}$ in the space surrounding such objects ${ }^{1}$.

Chromo-magnetic moments of quarks, originating from the color charge, are responsible for the hyperfine splitting of hadronic masses. Different orientations of constituent quark spins can change the interaction energy of quark chromo-magnetic moments in hadrons by hundreds $\mathrm{MeV}$. Typical examples are $\eta_{c}-\psi(1 S)$ or $N-\Delta$ mass differences. At the same time, ordinary magnetic moments of quarks also interact with each other inside hadrons. Being unconfined, dipole magnetic fields of quarks contribute to baryon magnetic moments, which respond to external magnetic fields. Typical examples are NMR experiments with protons or the precession of polarized hyperons [4].

Mesons, composed of quarks and antiquarks should (similarly to baryons) possess magnetic moments provided their angular momentum $J \neq 0$. We will show that magnetic moments of vector mesons are comparable in size to the magnetic moment of proton or neutron $\left(2.79 \mu_{N},-1.91 \mu_{N}\right)$. Due to the short lifetime of vector mesons $(c \tau<500 \mu \mathrm{m})$, precession of their spin in the magnetic field of laboratory magnets $(B<10$ Tesla) is not measurable, contrary to the case [4] of hyperons. However, in the magnetic fields $B \approx 10^{14}$ Tesla created in relativistic nucleus-nucleus collisions, electromagnetic structure of mesons may become important and have measurable consequences.

In this contribution we assume mesons to be composed of the constituent quark and anti-quark, both of them having its own static magnetic moment $\mu_{q}^{*}$ and $\mu_{\bar{q}}^{*}$. Response of QCD vacuum (chiral condensates) to strong magnetic fields [5] is not considered here.

\section{Magnetic moments of Quarks and Mesons}

Magnetic moments of hyperons and lightest baryons can be understood assuming the existence of the magnetic moment of constituent quarks. Indeed, dividing the low-mass $s=1 / 2$ baryons into categories: a) $q_{a}\left(q_{b} q_{a}\right)$ containing two quarks of the same flavor, and b) $q_{a}\left(q_{b} q_{c}\right)$ with all three quarks different, and observing that $s=3 / 2$ baryons have all quarks spins parallel, one can approximate magnetic moments of $p, n, \Sigma^{+}, \Sigma^{-}, \Xi^{o}, \Xi^{-}$baryons as $\mu=4\left(\mu_{a}^{*}-\mu_{b}^{*}\right) / 3$, moments of $\Omega^{-}, \Delta^{+}, \Delta^{++}$as $\mu=\sum \mu_{q_{i}}^{*}$ and for $\Lambda^{o}$ hyperon $\mu=\mu_{s}^{*}$. Convincing agreement with experimental data is obtained assuming $\mu_{u}^{*}=1.85 \mu_{N}, \mu_{d}^{*}=-0.97 \mu_{N}$, and $\mu_{s}^{*}=-0.61 \mu_{N}$, where $\mu_{N}$ is the nuclear magneton $\mu_{N}=5 \cdot 10^{-27} \mathrm{~J} / \mathrm{T}$.

From Dirac equation we know that elementary particles with spin $s=1 / 2$ and charge $Q$ should have magnetic moment $\mu=\hbar Q / 2 m c$, as can be shown by F-W transformation [3] of Dirac equation. Magnetic moments $\mu_{q}^{*}$ of constituent $u, d, s$ quarks are in agreement with equation $\mu^{*}=\hbar Q / 2 m c$,

\footnotetext{
${ }^{1}$ Kerr-Neuman solutions for charged rotating singular massive objects have gyromagnetic factor $g=2$ similarly to electron, and other speculations on relation between general theory of relativity and particle physics are known [6].
} 
if constituent quark masses $\left(m_{u}^{*} \approx m_{d}^{*} \approx 330 \mathrm{MeV}\right.$, and $\left.m_{s}^{*}=510 \mathrm{MeV}\right)$ are used for the mass $m$. One can predict $\mu_{c}^{*}=0.40 \mu_{N}, \mu_{b}^{*}=-0.066 \mu_{N}$ using $m_{c}^{*}=1550 \mathrm{MeV}$ and $m_{b}^{*}=4730 \mathrm{MeV}$.

Vector mesons are bound states of quark-antiquark with parallel spins. Magnetic moment of vector $\left(J^{P}=1^{-}\right)$mesons should then be calculable as $\mu=\sum \mu_{q_{i}}^{*}$, similarly to $s=3 / 2$ baryons $\Omega^{-}$, and $\Delta(1232)$. For charged vector mesons $\rho^{-}, K^{*+}, D^{*-}, D_{s}^{*-}$ and $B^{*-}$ one obtains $\mu^{*}=-2.82 \mu_{N}$, $2.46 \mu_{N},-1.37 \mu_{N},-1.02 \mu_{N}$, and $-1.92 \mu_{N}$, which agrees well with theoretical calculations [7]. For the neutral open-flavor vector mesons $B^{o *}, B_{s}^{o *}, K^{o *}$ and $D^{o *}$ one has $\mu^{*}=-0.9 \mu_{N},-0.5 \mu_{N}$, $-0.3 \mu_{N}$ and $-1.4 \mu_{N}$. These predictions are summarized in Table 1.

Table 1: Magnetic moment estimates for charged and neutral open-flavor $J^{P}=1^{-}$mesons.

\begin{tabular}{|c||c|c|c|c|c||c|c|c|c|}
\hline & $\rho^{-}$ & $K^{*+}$ & $D^{*-}$ & $D_{s}^{*-}$ & $B^{*-}$ & $B^{* o}$ & $B_{s}^{* o}$ & $K^{\circ *}$ & $D^{o *}$ \\
\hline$m[\mathrm{MeV}]$ & 770 & 892 & 2010 & 2112 & 5325 & 5325 & 5415 & 896 & 2007 \\
\hline$q \bar{q}$ & $d \bar{u}$ & $u \bar{s}$ & $d \bar{c}$ & $s \bar{c}$ & $b \bar{u}$ & $d \bar{b}$ & $s \bar{b}$ & $d \bar{s}$ & $c \bar{u}$ \\
\hline$\mu^{*}\left[\mu_{N}\right]$ & -2.82 & 2.46 & -1.37 & -1.02 & -1.92 & -0.9 & -0.5 & -0.3 & -1.4 \\
\hline
\end{tabular}

Magnetic interaction of hidden-flavor vector mesons $\mathrm{J} / \psi, \Upsilon, \varphi$ and of pseudoscalar mesons is however more complex. We describe it in the next section based on the similarity with Positronium or Muonium bound states. Interaction of QCD condensates with strong magnetic fields [5], which have influence on the meson properties, is neglected here.

\section{Positronium and Quarkonium in the Magnetic field}

Behavior of Positronium $\left(e^{+} e^{-}\right)$ground state in magnetic fields $(B \approx 1$ Tesla) has been investigated many years ago [8]. The interaction of external magnetic field $B$ with positron and electron magnetic moments $\mu_{e^{-}}=-\left|\mu_{e^{+}}\right|$leads to the quantum interference (mixing) of ortho-Positronium $\left(\mathrm{J}=1, m_{z}=0\right)$ state $\Psi_{o}=(\uparrow \downarrow+\downarrow \uparrow) / \sqrt{2}$ with para-Positronium state $\Psi_{p}=(\uparrow \downarrow-\downarrow \uparrow) / \sqrt{2}$. Two $\left(m_{z}= \pm 1\right)$ ortho-Positronium states $(\uparrow \uparrow)$ and $(\downarrow \downarrow)$ have zero magnetic moment, remain intact by the magnetic field, and decay into $3 \gamma, 5 \gamma$ with unchanged lifetime $\tau_{o}=1 / \lambda_{o}=1 \cdot 4 \cdot 10^{-7} \mathrm{~s}$. New mixed ortho-state $\Psi_{o}^{+}=\cos (\alpha) \Psi_{o}+\sin (\alpha) \Psi_{p}$ can decay also into $2 \gamma$ with the probability determined by the admixture of para-Positronium state in $\Psi_{o}^{+}$wave-function. As a consequence, the lifetime of $\Psi_{o}^{+}$state is strongly reduced and up to $33 \%$ of $3 \gamma$ decays of ortho-Positronium disappear [9] in the static magnetic field $(B \approx 1 \mathrm{~T})$. At the same time, para-Positronium state in the magnetic field gets admixture of ortho-state and new state $\Psi_{p}^{-}=\cos (\alpha) \Psi_{p}-\sin (\alpha) \Psi_{o}$ decays also into $3 \gamma$ besides its natural $2 \gamma$ and $4 \gamma$ annihilation channels. The original lifetime $\tau_{p}=1 / \lambda_{p}=1.2 \cdot 10^{-10} \mathrm{~s}$ of para-Positronium is only slightly increased. Decay rates of new mixed states $\Psi_{p}^{-}$and $\Psi_{o}^{+}$are [8]

$$
\lambda_{p}^{-}=\left(\lambda_{p}+y^{2} \lambda_{o}\right) /\left(1+y^{2}\right) \quad \lambda_{o}^{+}=\left(\lambda_{o}+y^{2} \lambda_{p}\right) /\left(1+y^{2}\right)
$$

and their energies depend on the magnetic field as

$$
E_{p}^{-}=\frac{1}{2} \Delta E_{h f}\left(1-\sqrt{1+x^{2}}\right) \quad E_{o}^{+}=\frac{1}{2} \Delta E_{h f}\left(1+\sqrt{1+x^{2}}\right)
$$

where $y=x /\left(1+\sqrt{1+x^{2}}\right)$ and $x=4 \mu_{e}^{\prime} B / \Delta E_{h f}$. (Here $\Delta E_{h f}=8.4 \cdot 10^{-4} \mathrm{eV}$ is hyperfine energy splitting of Positronium, and $\mu_{e}^{\prime}$ is the magnetic moment of bound electron [10]). The mixing parameter $\alpha$ in states $\Psi_{o}^{+}$and $\Psi_{p}^{-}$depends on the magnetic field as: $\sin (\alpha)=y / \sqrt{1+y^{2}}$. 
Fraction $F$ of all ortho-Positronium decays via original $(3 \gamma)$ channel is [8]: $F=2 / 3+1 / 3(1+R)$, where $R=y^{2}\left(\lambda_{p} / \lambda_{o}\right)$. For $B \approx 1$ T one has $x=0.27$ (using $\mu_{e}=5.79 \cdot 10^{-5} \mathrm{eV}$ ) and $y=0.12$. This gives $R=16.4$ and $F=2 / 3+0.02$ : almost all $\Psi_{o}^{+}$states decay via (originally C-parity violating) $2 \gamma$ channel.

Quarkonium $(c \bar{c}),(b \bar{b})$ in the ground state can be found in spin-triplet configuration known as vector mesons $\mathrm{J} / \psi, \Upsilon(9460)$ or in the spin-singlet state observed as pseudoscalars $\eta_{c}(2980)$, $\eta_{b}(9389)$. Due to chromo-magnetic hyperfine interaction of quarks, energies of para-Quarkonium $\left(\mathrm{J}=0^{-+}\right)$and ortho-Quarkonium $\left(\mathrm{J}=1^{--}\right)$are separated by $\Delta E_{h f}^{c \bar{c}}=116.4 \mathrm{MeV}$ and $71.4 \mathrm{MeV}$ for $(b \bar{b})$.

Magnetic moments $\mu_{c}, \mu_{b}$ of quarks are small compared to $\mu_{e}$, however they can interact with the external magnetic field. Hamiltonian term $\mathscr{H}_{i}=\mu_{q} B\left[\sigma_{z}(q)-\sigma_{z}(\bar{q})\right]$ will induce mixing of two orthogonal $m_{z}=0$ quantum states similarly to Positronium. Thus, in a sufficiently strong magnetic fields (e.g. in heavy ion collisions) one can anticipate that quantum mixing of ortho- and paraQuarkonium happens (quantum numbers of $q \bar{q}$ states correspond to Positronium). The mixing of states is controlled by simple parameter $x=4 \mu_{q} B / \Delta E_{h f}^{q \bar{q}}$ in Eq.(3.2). For the Positronium $x=0.27$ in magnetic field $B=1$ Tesla. This is achieved for $B=6 \cdot 2 \cdot 10^{14} \mathrm{~T}$ in the case of Charmonium and for $B=2.3 \cdot 10^{15}$ Tesla for the Bottomium ground states.

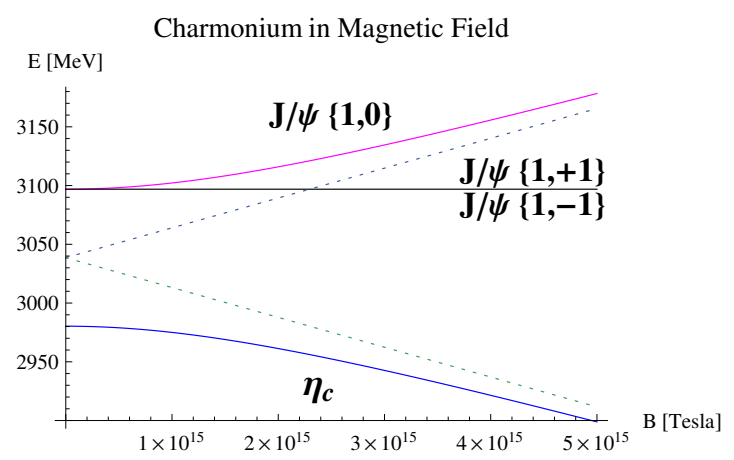

Figure 1: Energy of $\eta_{c}$ and $J / \Psi$ in magnetic field.

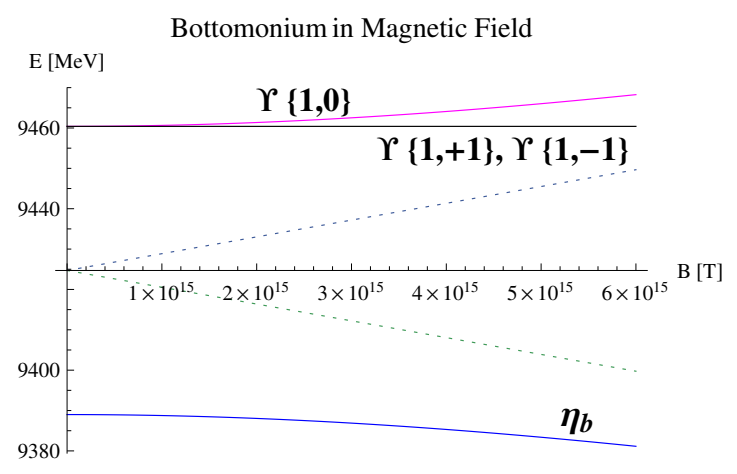

Figure 2: Energy of $\eta_{b}$ and $\Upsilon$ in magnetic field.

Energy (mass) of para-Charmonium state $\tilde{\eta}_{c}$ is lowered according to Eq.(3.2) by $2 \mathrm{MeV}$ for $B=6 \cdot 10^{14}$ Tesla, while its decay width $\lambda_{p}=26.7 \mathrm{MeV}$ is not affected significantly (see Eq.3.1). Similarly to ortho-Positronium, mixed $(q \bar{q})$ state $\Psi_{o}^{+}$is allowed to decay via new channels. For example, $\mathrm{J} / \psi \rightarrow \operatorname{ggg}$ decay normally happens in $64 \%$ of cases, but in the case of quantum mixing $\Psi_{o}^{+} \rightarrow g g$ is possible, which increases its decay width $\lambda_{o}=93 \mathrm{keV} \rightarrow 527 \mathrm{keV}$ at $B=6 \cdot 10^{14} \mathrm{~T}$ (see Eq.3.1). This means reduction of $\Psi_{J / \psi}^{+}$lifetime by factor 5x. Similar behavior can be expected for $\psi(2 S)$ coupled to $\eta_{c}(2 S)$ with $\Delta E_{h f}^{c c}=47 \mathrm{MeV}$ and for the Bottomium states $\Upsilon(1 S)$ and $\eta_{b}(9391)$. Assuming $^{2} \lambda_{p}=\Gamma_{\eta_{b}} \approx 10 \mathrm{MeV}$ in Eq.3.1, one has $\lambda_{o}=54 \mathrm{keV} \rightarrow 102 \mathrm{keV}$ in the static magnetic field $B=1.2 \cdot 10^{15} \mathrm{~T}$, reducing the lifetime of mixed state $\Psi_{\Upsilon}^{+}$by factor $2 \mathrm{x}$. Mass of $\left(m_{z}=0\right)$ vector state $\Psi_{\Upsilon}^{+}$increases according to Eq.3.2 by $1 \mathrm{MeV}$ at $2 \cdot 10^{15} \mathrm{~T}$ and therefore it should experience repulsive (diamagnetic) interaction in the magnetic field.

The above mentioned modifications of heavy Quarkonium properties are of purely electromagnetic origin. Changes due to gluonic or quark condensates [11] in hot QCD medium (which are influenced also by strong magnetic fields [12]) are not considered here.

\footnotetext{
${ }^{2}$ Decay width of $\eta_{b}$ is only estimated [13], experimental verification is needed.
} 


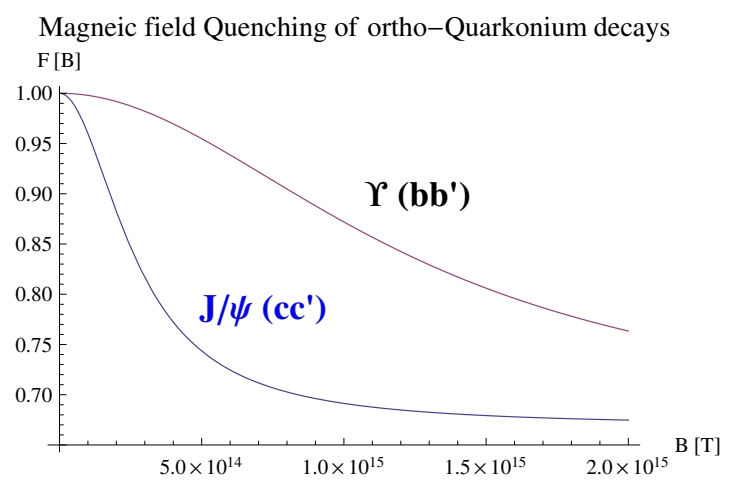

Figure 3: Fraction of $J / \Psi$ and $\Upsilon$ particles decaying into original $\left(l^{+} l^{-}\right)$channels in the magnetic field.

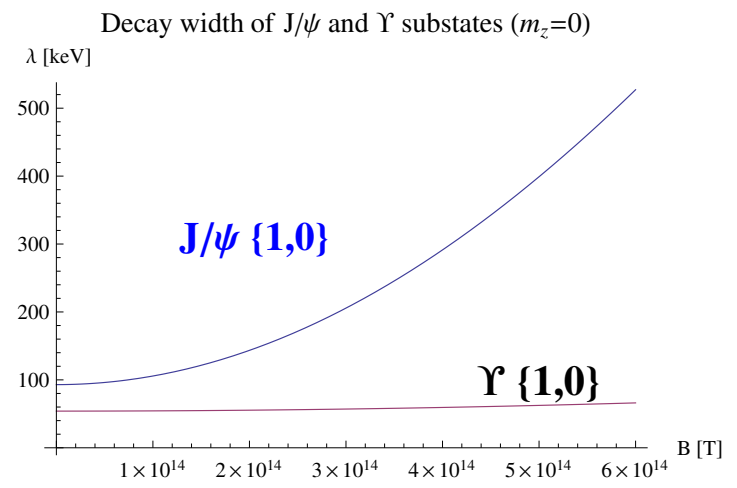

Figure 4: The increase of decay widths for $\Psi_{J / \psi}^{+}$ and $\Psi_{\Upsilon}^{+}$states $\left(m_{z}=0\right)$ in the magnetic field.

\section{Dilepton signatures of Quarkonium states mixing in the magnetic field}

In the strong magnetic field $\left(B=6 \cdot 10^{14}\right.$ Tesla), new ortho-Charmonium state $\Psi_{o}^{+}$can decay also via 2-gluon $(\mathrm{gg})$ channel due to the admixture of pseudoscalar $\Psi_{p}=\eta_{c}$ state in its wave function. Therefore, a significant fraction of hadronic $(g g g, g g \gamma)$ and leptonic $\left(e^{+} e^{-}, \mu^{+} \mu^{-}\right)$decays of new $\Psi_{o}^{+}$state will disappear, being replaced by the competing $(g g)$ decays. $R=y^{2}\left(\lambda \eta_{c} / \lambda^{\psi}\right)=4.13$ for $y=0.12, x=0.27$, and thus [8] $(1-F)=R / 3(1+R)=26.8 \%$ of dilepton decays of all $\mathrm{J} / \psi$ mesons in the static magnetic field $B=6 \cdot 10^{14}$ Tesla should be quenched (see Fig.3) analogously to Positronium [9]. Those missing dilepton pairs belong to $\Psi_{o}^{+}$mixed state, for which the energy (see Fig.1) is increased by $\delta E=\sqrt{1+x^{2}}\left(\Delta E_{h f}^{c \bar{c}} / 2\right)$, compared to two $\left(m_{z}= \pm 1\right)$ states J/ $\Psi$. Therefore, just above the observed $\mathrm{J} / \Psi$ peak in the measured dilepton mass spectrum, those missing $\left(l^{+} l^{-}\right)$ pairs might produce a little dip after the subtraction of Gaussian $\mathrm{J} / \Psi$ peak at $3096 \mathrm{MeV}$ from the experimentally measured dilepton mass spectrum.

At the same time, new mixed para-Charmonium state $\Psi_{p}^{-}=\tilde{\eta}_{c}$ can decay into dilepton pairs, proportionally to the admixture of ortho-state $\Psi_{o}\left(m_{z}=0\right)$ in its wave function. A small bump below $\mathrm{J} / \psi$ mass can appear in the observed dilepton spectrum due to dileptons originating from $\tilde{\eta}_{c} \rightarrow l^{+} l^{-}$decays in the magnetic field. Dilepton pairs originating from $\tilde{\eta}_{c} \rightarrow l^{+} l^{-}$decays can be produced only in extreme magnetic fields, which disappear soon when partonic plasma expands. Since lifetime of $\tilde{\eta}_{c}$ and $\tilde{\eta}_{b}$ quarkonium states is almost unchanged in the magnetic fields (see Eq.3.1), total yield of such anomalous dilepton pairs depends on the ratio $\tau_{B} / \tau_{\eta_{c}}$ of the magnetic field lifetime $\tau_{B}$ and the lifetime of $\tilde{\eta}_{c}$. For bottomiun states $\Upsilon(1 \mathrm{~s}), \Upsilon^{\prime}(2 \mathrm{~s})$ and light vector mesons $\left(\varphi, \omega, \rho^{o}\right)$ one may expect similar effects to happen.

\section{Open-flavor mesons in magnetic field}

The interaction of heavy flavor mesons containg quarks of different mass, for example $(b \bar{d})=B^{+}$ or $(c \bar{u})=D^{o}$, with magnetic fields can be understood using the analogy with Muonium $\left(\mu^{+} e^{-}\right)$. Spin-triplet Muonium ground state [14] corresponds to $J=1$ open-flavor mesons, e.g. $B^{o *}(5325)$, $D^{o *}(2007)$, while para-Muonium (spin-singlet) state corresponds to pseudoscalar $(J=0)$ mesons 
$B^{o}, D^{o}, B_{s}^{o}$. Similarly to Muonium, magnetic moments of the constituents are different $\left|\mu_{1}\right| \neq\left|\mu_{2}\right|$ and therefore $\left(m_{z}= \pm 1\right)$ ortho-states $|\uparrow \uparrow\rangle$ and $|\downarrow \downarrow\rangle$ have magnetic moment $\vec{\mu}_{12}=\vec{\mu}_{1}+\vec{\mu}_{2}$. For example, $B^{+*}$ magnetic moment is $\mu_{B^{*+}} \approx\left|\mu_{u}\right|+\left|\mu_{\bar{b}}\right|=1.92 \mu_{N}$, for $B^{-*}$ meson $\mu_{B^{*-}} \approx-1.92 \mu_{N}$ (compare to neutron $\mu_{n}=-1.91 \mu_{N}$ ) and for $D^{o *}$ meson one has: $\mu_{D^{o *}} \approx\left|\mu_{c}\right|-\left|\mu_{\bar{u}}\right|=-1.4 \mu_{N}$.

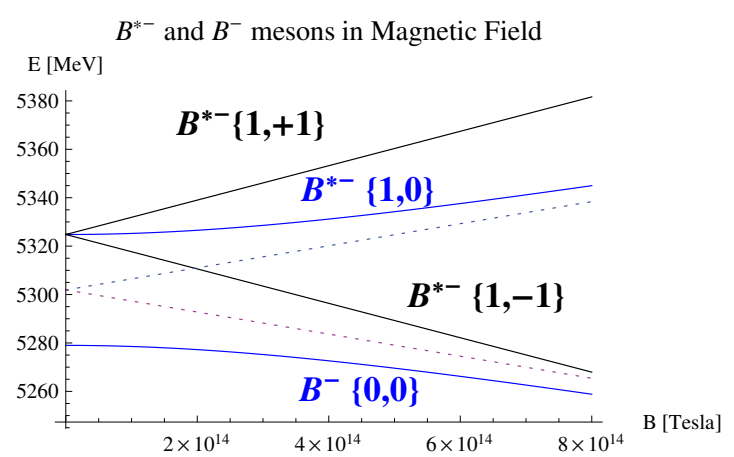

Figure 5: Energy of $B^{-}$and $B^{-*}$ in magnetic field.

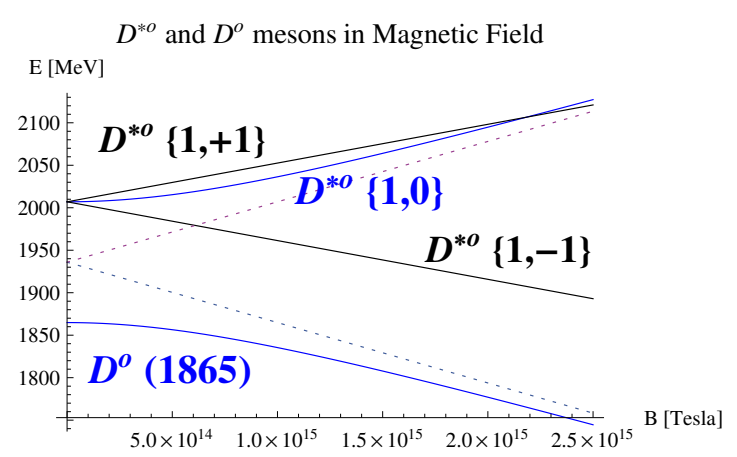

Figure 6: Energy of $D^{o}$ and $D^{o *}$ in magnetic field.

Two $\left(m_{z}=0\right)$ states $\Psi_{p}=(\uparrow \downarrow-\downarrow \uparrow) / \sqrt{2}$ and $\Psi_{o}=(\uparrow \downarrow+\downarrow \uparrow) / \sqrt{2}$ have zero magnetic moment similarly to Positronium and Quarkonium. Electromagnetic interaction of mesons with external magnetic field is described by Hamiltonian term $\hat{\mathscr{H}}_{i}=-B \cdot \hat{\mu}_{q \bar{q}}$. For charged meson $B^{-}=(b \bar{u})$ we have $\hat{\mu}_{q \bar{q}}=-\left|\mu_{b}\right| \hat{\sigma}_{z}-\left|\mu_{\bar{u}}\right| \hat{\sigma}_{z}$ and for neutral $(c \bar{u})=D^{o}$ meson: $\hat{\mu}_{q \bar{q}}=\left|\mu_{c}\right| \hat{\sigma}_{z}-\left|\mu_{\bar{u}}\right| \hat{\sigma}_{z}$. In the magnetic field, quantum interference (mixing) of $\Psi_{p}$ and $\Psi_{o}$ states takes place similarly to Muonium [14] or Positronium [8]. New mixed states $\Psi_{o}^{+}$and $\Psi_{p}^{-}$can be expressed as

$$
\Psi_{o}^{+}=\frac{c_{\alpha}+s_{\alpha}}{\sqrt{2}}|\uparrow \downarrow\rangle+\frac{c_{\alpha}-s_{\alpha}}{\sqrt{2}}|\downarrow \uparrow\rangle \quad \Psi_{p}^{-}=\frac{c_{\alpha}-s_{\alpha}}{\sqrt{2}}|\uparrow \downarrow\rangle-\frac{c_{\alpha}+s_{\alpha}}{\sqrt{2}}|\downarrow \uparrow\rangle
$$

where $c_{\alpha}=1 / \sqrt{1+y^{2}}$ and $s_{\alpha}=y / \sqrt{1+y^{2}}$, while $y=x /\left(1+\sqrt{1+x^{2}}\right)$ and $x=2 \mu_{12} B / \Delta E_{h f}$. As the magnetic field increases, the states $\Psi_{o}^{+}$and $\Psi_{p}^{-}$"rotate" by mixing angle $\alpha=\arctan (y)$ as shown in Fig.7. For very large magnetic fields angle $\alpha$ approaches maximum value $\alpha \rightarrow \frac{\pi}{2}$ and (using Eq.5.1) we have $\Psi_{o}^{+}=|\uparrow \downarrow\rangle$ and $\Psi_{p}^{-}=-|\downarrow \uparrow\rangle$. In such extreme case the magnetic moment $\langle\hat{\mu}\rangle$ of spin-singlet $D^{o}$ meson becomes $\left\langle\downarrow \uparrow\left|\hat{\mu}_{q \bar{q}}\right| \downarrow \uparrow\right\rangle=\left|\mu_{c}\right|+\left|\mu_{u}\right|$. Thus, induced magnetic moment of $D^{o}$ meson can be larger than magnetic moment of $\left(m_{z}= \pm 1\right)$ states of $D^{o *}$, which corresponds to the behavior of para- and ortho-states of Muonium [14]. For intermediate magnetic fields

$$
\left\langle\Psi_{p}^{-}\left|\hat{\mu}_{c \bar{u}}\right| \Psi_{p}^{-}\right\rangle=\left(\left|\mu_{c}\right|+\left|\mu_{u}\right|\right) \sin (2 \alpha) \quad, \quad\left\langle\Psi_{o}^{+}\left|\hat{\mu}_{c \bar{u}}\right| \Psi_{o}^{+}\right\rangle=-\left(\left|\mu_{c}\right|+\left|\mu_{u}\right|\right) \sin (2 \alpha)
$$

where $\sin (2 \alpha) \rightarrow 1$ for $B \rightarrow \infty$. The intrinsic magnetic polarizability [15] of meson, originating from the mixing of singlet and the lowest triplet $\left(m_{z}=0\right)$ states, is $\beta_{M}^{i n t}=2\left\langle\Psi_{p}^{-}\left|\hat{\mu}_{q \bar{q}}\right| \Psi_{o}^{+}\right\rangle^{2} / \Delta E_{h f}$. This gives $\beta_{M}^{\text {int }}=2\left(\left|\mu_{c}\right|+\left|\mu_{u}\right|\right)^{2} / \Delta E_{h f}^{c \bar{u}}$ for $D^{o}$ and $\bar{D}^{o}$ mesons. For charged $B^{-}$mesons the magnetic polarizability is $\beta_{M}^{\text {int }}=2\left(\left|\mu_{b}\right|-\left|\mu_{u}\right|\right)^{2} / \Delta E_{h f}^{b \bar{u}}$. Maximal induced magnetic moment of $B^{-}$meson $\left\langle\uparrow \downarrow\left|\hat{\mu}_{b \bar{u}}\right| \uparrow \downarrow\right\rangle=\left|\mu_{\bar{u}}\right|-\left|\mu_{b}\right|$, which cannot exceed magnetic moment of $B^{-*}$ state: $\mu=\left|\mu_{b}\right|+\left|\mu_{u}\right|$.

Open-flavor neutral and charged mesons do not annihilate into $\gamma \gamma, g g$ or $3 \gamma, g g g$. Vector states $D^{*}, B^{*}$ usually decay into the spin-singlet configurations by the emission of $\gamma, \pi^{o}$, or $\pi^{ \pm}$. For example, $D^{*+}(2010)$ meson $\left(\Gamma=96 \pm 22 \mathrm{keV}, \tau \approx 7 \cdot 10^{-21}\right.$ s) decays in $68 \%$ of cases into $D^{o}+\pi^{+}$, 


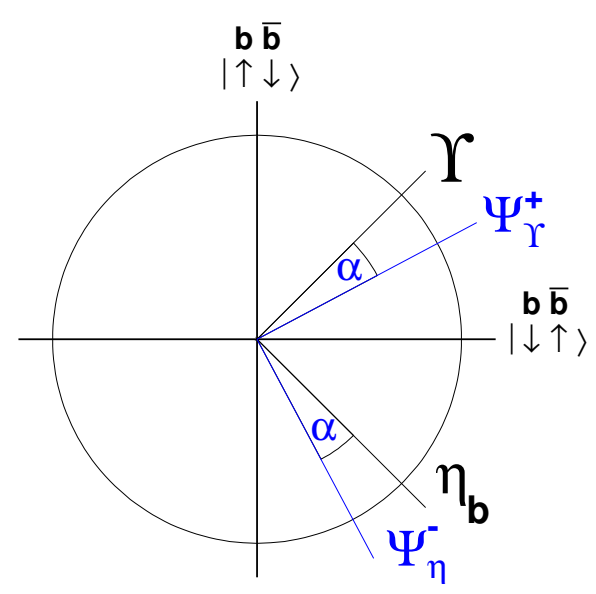

Figure 7: Rotation of new states $\Psi_{\Upsilon}^{+}$and $\Psi_{\eta_{b}}^{-}$ relative to $\Upsilon\left(m_{z}=0\right)$ and $\eta_{b}$ in magnetic field.

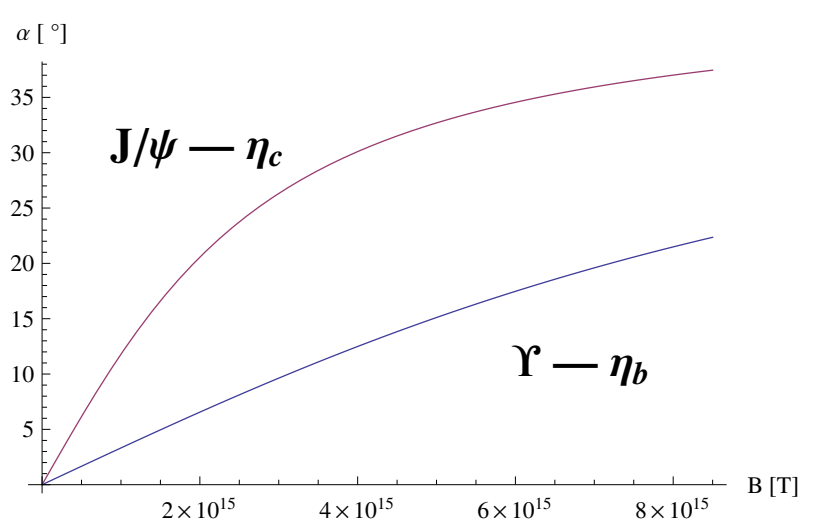

Figure 8: Angle $\alpha$ for the mixing of quarkonium states in strong magnetic fields: $\tan (\alpha)=x /\left(1+\sqrt{1+x^{2}}\right)$.

while its long-lived spin-singlet partner $D^{+}\left(\tau \approx 10^{-12}\right.$ s) contributes to $e^{+}, \mu^{+}$lepton spectra in heavy ion collisions. Hyperfine splitting in $D^{+}, D^{*+}$ system is $\Delta E_{h f}=140 \mathrm{MeV}$, and $\mu_{D^{*+}} \approx 1.37 \mu_{N}$, ratio $\tau_{D^{+*}} / \tau_{D^{+}} \approx 7 \cdot 10^{-9}$. If the magnetic field $B \approx 10^{15}$ Tesla lasts for a sufficiently long time, quantum superposition of $D^{+}$meson with $\left(m_{z}=0\right)$ ortho-state $D^{*+}(2010)$ can occur. However, due to the mass (energy conservation) restriction, the mixed $\Psi_{D^{+}}^{-}$para-state cannot decay into $D^{o}+\pi^{+}$. Therefore, we do not expect consequences of the quantum mixing in large magnetic fields to be significant in this case.

\section{The case of $\varphi(1020)$ and $\omega, \rho$ mesons}

Similarly to the case of Charmonium and Bottomium described above, one may expect that $\varphi(1020)$ meson ( $s \bar{s}$ state with $J=1)$ should have a spin-singlet $(s \bar{s} J=0)$ partner, which is lighter compared to $\varphi$ due to the hyperfine interaction of quark chromo-magnetic moments. Pure $\eta_{s}$ state is however not identified in the mass spectrum of hadrons. The only reasonable partner for $\varphi$ is $\eta^{\prime}(958)$. We have $\Delta M\left(\Upsilon-\eta_{b}\right)=71.4 \mathrm{MeV}$ and $\Delta M\left(J / \psi-\eta_{c}\right)=116.6 \mathrm{MeV}$, which agrees with chromo-magnetic moments of heavy $b, c$ quarks to be inversely proportional to the constituent quark masses. In the sense of Rújula-Georgi-Glashow [16] picture of mesons, one can proceed to the light mass region and observe: $\Delta M(\omega-\eta(547))=234.8 \mathrm{MeV}$ and $\Delta M(\rho-\pi)=640 \mathrm{MeV}$. The mass splitting of $\Delta M\left(\varphi-\eta_{s}\right)$ should then be located inbetween $116 \mathrm{MeV}$ and $235 \mathrm{MeV}$. However, $\Delta M\left(\varphi-\eta^{\prime}(958)\right)=61.7 \mathrm{MeV}$. This is too small if chromo-magnetic moment of $s$-quark is inversely proportional to its constituent mass. Clearly, mass of $\eta^{\prime}(958)$ meson has a contribution of different origin [17] if compared to heavy Quarkonium states $\eta_{b}, \eta_{c}$.

Nevetherless, let us assume $\eta^{\prime}(958)$ to be the mixing partner (e.g. with $66 \% s \bar{s}$ content) for $\varphi$. The mass of $\varphi$ state with $\left(m_{z}=0\right)$ is then expected to increase (see Fig.9) in the magnetic field, while $\varphi\left(m_{z}= \pm 1\right)$ states remain unaffected. At the same time, mass of pseudoscalar $K^{ \pm}$mesons $(s \bar{u})$ and $K^{o}$ mesons $(d \bar{s})$ is expected to decrease (see Fig.10), due to $K-K^{*}$ quantum mixing in the magnetic field. 
Such changes of $\varphi$ and $K$ meson masses will enhance phase-space for $\varphi \rightarrow K K$ decays. It is interesting to observe that due to different magnetic polarizabilities, mass decrease of $K^{ \pm}$and $K^{o}$ mesons in the magnetic field is significantly different. Our preliminary estimate for the static field $B=10^{15}$ Tesla gives $\Delta M_{K^{ \pm}}=-3.8 \mathrm{MeV}$ and $\Delta M_{K^{o}}=-14.6 \mathrm{MeV}$. Decays $\phi \rightarrow K^{+} K^{-}$and $\phi \rightarrow K^{o} \bar{K}^{o}$ are competing with each other with similar probabilities: $P_{K_{ \pm}}=48.9 \%$ and $P_{K_{ \pm}}=34.2 \%$. Larger enhancement of phase-space for $\phi \rightarrow K^{o} \bar{K}^{o}$ means less $\phi \rightarrow K^{+} K^{-}$decays than expected. Since dilepton channel $\phi \rightarrow l^{+} l^{-}$is not affected by the change of $K$ meson masses, ratio $\Gamma_{l^{+} l^{-}} / \Gamma_{K^{+} K^{-}}$gets modified in strong magnetic fields. This may be related to subtle differencies in $p_{t}$ slopes and total yields of $\varphi$ mesons observed via $l^{+} l^{-}$and $K^{+} K^{-}$decays in heavy ion collisions at SPS [18] and RHIC [19]. All changes in $\varphi$ and $K$ meson masses considered here are of purely electromagnetic origin. Taking into account also QCD effects can modify the situation very substantially.

A possibility of mixed $\Psi_{\eta^{\prime}}^{-}=\cos (\alpha) \eta^{\prime}+\sin (\alpha) \varphi$ state to decay into $\rightarrow K^{+} K^{-}$is forbidden due to the mass (energy) constraints: $M_{\eta^{\prime}}<2 M_{K^{ \pm}}$. Additionally, mass of $\eta^{\prime}$ is expected to decrease significantly in dense QCD environment [20]. Decays of state $\Psi_{\eta^{\prime}}^{-}$directly to $\rightarrow l^{+} l^{-}$are possible proportionally to the admixture of $\left(m_{z}=0\right) \varphi$ meson ortho-state in the $\Psi_{\eta^{\prime}}^{-}$wave function. Spectrum of dileptons below $\varphi$ meson mass may get contaminated by such dileptons.

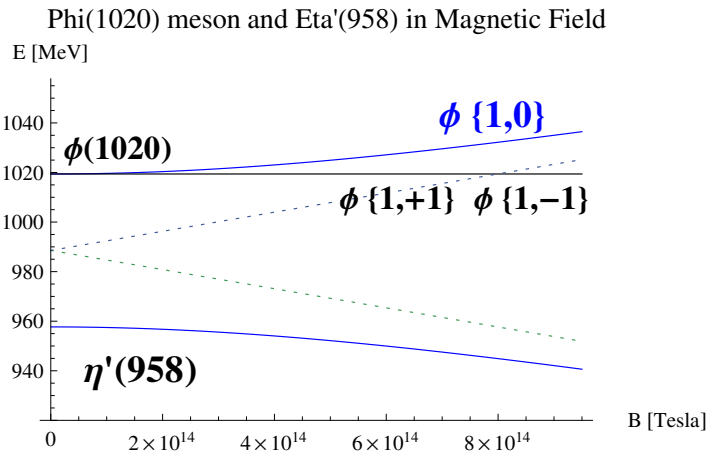

Figure 9: Energy of $\varphi(s \bar{s})$ and $\eta_{s}^{\prime}$ in magnetic field.

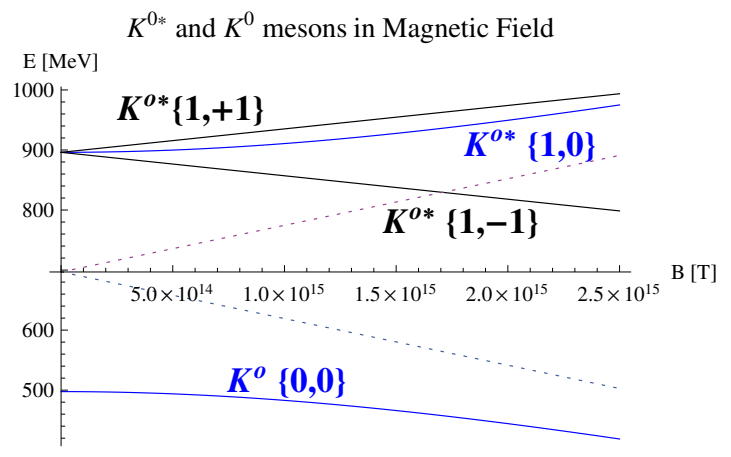

Figure 10: $K^{o}$ and $K^{o *}$ mesons in magnetic field.

One may also speculate that in the strong magnetic fields created in heavy-ion collisions a mixing of para and ortho $\left(m_{z}=0\right) q \bar{q}$ states may happen also for light mesons $\omega, \rho^{o}, \eta, \pi^{o}$. Such mixing would mean that $\eta(547)$, for example, would become a mixed $\Psi_{\eta}^{-}$state, being able to decay into dilepton pairs with invariant masses in the region $400-550 \mathrm{MeV}$. If mixing of $\pi^{o}-\rho^{o}$ states takes place, then dilepton spectrum can become contaminated via direct decays of mixed state $\Psi_{\pi^{o}}^{-} \rightarrow e^{+} e^{-}$. In such preliminary considerations, one should take into account the lifetimes of $\rho^{o}(770), \omega(782)$ and $\eta(547)$ mesons $\left(\Gamma_{\rho}=149 \mathrm{MeV}, \Gamma_{\omega}=8.5 \mathrm{MeV}\right.$ and $\left.\Gamma_{\eta}=1.3 \mathrm{keV}\right)$. Mesons $\omega$ and $\eta$ decay outside the expanding fireball, when magnetic fields already disappeared. However, $\rho^{o}$ meson decays early, and $\rho^{o}-\pi^{o}$ superposition may have significant measurable effects.

Masses of light pseudoscalar mesons are expected to be modified also due to instanton-induced effects in dense QCD medium [20]. Non-trivial interplay of singlet-triplet states mixing in the magnetic field and flavor-mixing effects [20] may possibly take place. The mechanism of dilepton production, originating from the quantum mixing of states in magnetic field, may alternatively contribute to the usual explanation [21] of the excess of di-electron pairs below $\rho$ meson mass. 


\section{Summary and conclusions}

We have discussed the interaction of constituent quark magnetic moments with large magnetic fields created in heavy ion collisions. For the open-flavor vector mesons, magnetic moments are estimated, and for pseudoscalar mesons the magnetic polarizability is assessed.

Based on the analogy with Positronium behavior [8] in the magnetic field, we suggest that quantum superposition of spin-singlet and spin-triplet $\left(m_{z}=0\right)$ states of Quarkonium can also take place. Magnetic field quenching of ortho-Quarkonium is predicted to happen in the static magnetic fields comparable in strength to those created in heavy ion collisions.

If magnetic field quenching of ortho-Quarkonium takes place, $\mathrm{J} / \psi$ mesons in $m_{z}=0$ quantum substate may decay rapidly via new open channels ( $g g$, for example) due to the mixing with $\eta_{c}$ state. Substantial fraction (up to 30\%) of $\mathrm{J} / \psi$ mesons can thus dissapear from dilepton spectrum if strong magnetic fields last for sufficiently long time. This can directly influence one of the main signatures of deconfinement: total yield of $\mathrm{J} / \psi$ mesons [22] in the nucleus-nucleus collisions.

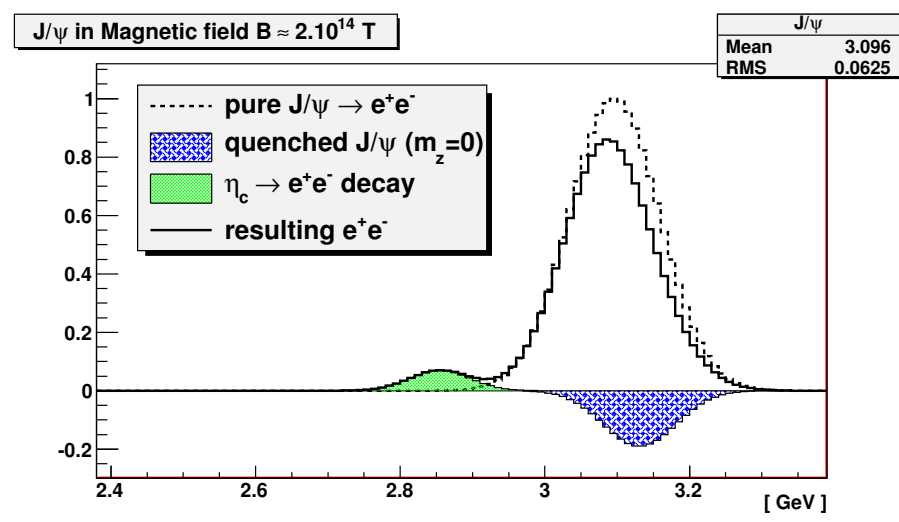

Figure 11: Dilepton spectrum modification for $\mathrm{J} / \Psi$ mesons decaying in strong magnetic field.

Energy of the mixed ortho-Charmonium $\left(m_{z}=0\right)$ state $\Psi_{J / \psi}^{+}$increases in the magnetic field when compared to $J / \psi(3096)$ particles, and missing dilepton pairs from quenched $\Psi_{J / \psi}^{+}$decays slightly modify dilepton spectrum (see Fig.11). New para-Charmonium state $\Psi_{\eta_{c}}^{-}$can decay to dilepton $l^{+} l^{-}$pairs directly (in the magnetic field) and little bump below $\mathrm{J} / \psi$ peak may appear.

For bottomium $\Upsilon$ and $\varphi(s \bar{s})$ states, dilepton signatures of the quantum mixing can be similar to $\mathrm{J} / \psi$. Since direction of the magnetic field created in heavy ion collisions is directly dependent on the reaction plane orientation, the azimuthal distribution of dilepton pairs from quenched quarkonium decays can be azimuthally asymmetrical, modifying the elliptic flow of vector-meson Quarkonium states J/ $\psi, \Upsilon$ and $\varphi$. For $\varphi$ meson, the modification of ratio $\Gamma_{l^{+} l^{-}} / \Gamma_{K^{+} K^{-}}$of $\varphi$ decay widths due to the changes of $K^{+}$and $K^{o}$ masses in strong magnetic fields may also take place.

Our simple predictions are obtained while ignoring QCD effects, e.g. the dependence of hadron masses on quark condensates [11] in the dense QCD medium.

We suggest that more precise and quantitative analysis of the phenomena discussed is needed. 


\section{Acknowledgements}

Special thanks are expressed here to H.G.Ritter for his support and to $\mathrm{Nu} \mathrm{Xu}$ for the kind invitation and the possibility to present talk at CPOD 2013 conference. The author thanks also to his colleagues at Comenius University (P.Bóna and V.Balek) in Bratislava, for a discussion on the specific issues of quantum state mixing phenomena. This work has been supported by Slovak Grant Agency VEGA under contract N. 1/0171/11.

\section{References}

[1] W.T.Deng and X.G.Huang, Phys.Rev. C85 (2012) 044907.

[2] D.E. Kharzeev, L.D. McLerran, H.J. Warringa, Nucl. Phys. A803 (2008) 227.

[3] L.L. Foldy and S.A. Wouthuysen, Physical Review 78 (1950) 29.

[4] C. Wilkinson et al., Phys. Rev. Lett. 58 (1987) 855.

[5] G.S. Bali et al, Phys. Rev. D86 (2012) 071502(R).

[6] B. Carter, Phys. Rev. 174 (1968) p.1559; M.J. Hadley, Europhysics Letters 95 (2011) 21003.

[7] F.T. Hawes and M.A. Pichowski, Phys. rev. C59 (1999) 1743. F.X. Lee, S. Moersbacher and W. Wilcox, PoS (Lattice 2007) 151.

[8] A.Rich, Rev. Mod. Phys. 53 (1981) p.127; O.Halpern, Phys. Rev. 94 (1954) p.904.

[9] V.W.Hughes, S.Marder and C.S.Wu, Physical Review 98 (1955) p.1840.

[10] A.P.Mills, Phys. Rev. A41 (1990) 502; A.Rich, Phys. Rev. A23 (1981) 2747.

[11] C.A.Dominguez, M. Loewe, J.C.Rojas and Y.Zhang, Phys. Rev. D83 (2011) 034033.

[12] R.Gatto and M.Ruggieri, Phys. Rev. D83 (2011) 034016.

[13] J.Beringer et al. (Particle Data Group), Physical Review D86 (2012) p. 1224.

[14] K.P-Jungmann, Proceedings of the Symposium in Honor of V.W.Hughes (Yale, 2003), World Scientific (2004) p.134.

[15] V.Bernard and D.Vautherin, Phys. Rev. D40 (1989) p. 1615.

[16] A.De Rújula, H.Georgi and S.L. Glashow, Physical Review D12 (1975) 147.

[17] R.Alkofer, C.S.Fischer and R.Williams, Eur. Phys. Jour. A38 (2008) p.53.

[18] M. Floris for NA60 Coll., Jour. Phys. G: Nucl. Part. Phys. 35 (2008) 104054; D.Adamová et al. (CERES Coll.), Phys. Rev. Lett. 96 (2006) 152301.

[19] V.Ryabov for PHENIX Coll., Jour. Phys. G: Nucl. Part. Phys. 35 (2008) 044030; D.Sharma for PHENIX Coll., Jour. Phys. G: Nucl. Part. Phys. 36 (2009) 064023.

[20] M.Takaziva, K.Tsushima, Y.Kohyama and K.Kubodera, Nucl. Phys. A507 (1990) p.611.

[21] G.E.Brown and M.Rho, Phys. Rev. Lett. 66 (1991) p. 2720; B.Schenke and C.Greiner, Phys. Rev. Lett. 98 (2007) 022301.

[22] T.Matsui and H.Satz, Phys. Lett. B 178 (1986) 416. 